\title{
Sensitivity of hip tissues contact evaluation to the methods used for estimating the hip joint center of rotation
}

\author{
Ehsan Arbabi • Jerome Schmid • Ronan Boulic • \\ Daniel Thalmann $\cdot$ Nadia Magnenat-Thalmann
}

Received: 4 August 2011 / Accepted: 7 February 2012/Published online: 29 February 2012

(C) International Federation for Medical and Biological Engineering 2012

\begin{abstract}
Computer-based simulations of human hip joints generally include investigating contacts happening among soft or hard tissues during hip movement. In many cases, hip movement is approximated as rotation about an estimated hip center. In this paper, we investigate the effect of different methods used for estimating hip joint center of rotation on the results acquired from hip simulation. For this reason, we use three dimensional models of hip tissues reconstructed from MRI datasets of 10 subjects, and estimate their center of rotation by applying five different methods (including both predictive and functional approaches). Then, we calculate the amount of angular and radial penetrations that happen among three dimensional meshes of cartilages, labrum, and femur bone, when hip is rotating about different estimated centers of rotation. The results indicate that hip simulation can be highly affected
\end{abstract}

E. Arbabi $(\bowtie) \cdot$ R. Boulic $\cdot$ D. Thalmann

Virtual Reality Lab, École Polytechnique Fédérale de Lausanne

(EPFL), Lausanne, Switzerland

e-mail: ehsan.arbabi@a3.epfl.ch; earbabi@ut.ac.ir

E. Arbabi

School of Electrical and Computer Engineering,

College of Engineering, University of Tehran,

North Kargar Avenue, Tehran, Iran

J. Schmid · N. Magnenat-Thalmann

MIRALAB, CUI, University of Geneva, Battelle 7,

route de Drize, Carouge, 1227 Geneva, Switzerland

R. Boulic

École Polytechnique Fédérale de Lausanne (EPFL), SCI, IC, RB, INJ 141, Station 14, 1015 Lausanne, Switzerland

D. Thalmann

Institute for Media Innovation, Nanyang Technological

University, 50 Nanyang Drive, Singapore, Singapore by the method used for estimating hip center of rotation. However, under some conditions (e.g. when Adduction or External Rotation are considered) we can expect to have a more robust simulation. In addition, it was observed that applying some methods (e.g. the predictive approach based on acetabulum) may result in less robust simulation, comparing to the other methods.

Keywords Hip simulation - Penetration depth - Center of rotation - Biomechanics

\section{Introduction}

Recently, many computer-based biomedical simulations have been developed in order to improve the speed and accuracy of medical treatments or diagnoses [1, 12, 16, 29]. Due to the increased attention received by hip problems in the orthopedic literatures [13, 23, 24], computer-based simulations of human hip joints have been investigated too.

In general, hip simulations include investigating contacts happening among soft or hard tissues during hip movements. Therefore, three dimensional virtual models of real tissues are created based on CT or MR images, and the virtual tissues that penetrate one another during the movement are simulated. In reality, stresses corresponding to the amount of virtual penetration occur to avoid such inter-penetration of real tissues. Therefore, having an estimation of penetration depth can give an appropriate measure for investigating hip contacts during the simulation $[1,2,14-16,26]$.

Since many hip simulations are based on rotation, it is required to have an estimation of hip joint center of rotation (HJC) $[1,15,16]$. There are several methods for estimating HJC [15], which may provide different values 
for the same hip model. Thus, it is essential to know how the results of hip simulations may vary based on the methods used for estimating HJC.

In this paper, we investigate the sensitivity of hip simulation to the location of the estimated center of rotation. We estimate HJC by five different methods for 10 patients. For each patient and each estimated HJC, we rotate the hip about different clinical axes (i.e. Adduction, Abduction, Internal Rotation, External Rotation, Flexion and Extension) and estimate changes in the penetration depth among hip tissues (including bone, cartilage and labrum), during the movement. Since our goal is to check the HJC sensitivity during 3D computer-based simulations, our estimations are done using 3D meshes reconstructed from MRI rather than using physical models or cadaver [21].

The results of this investigation can help researchers and clinicians by showing the impact of the applied HJC estimation methods on 3D hip simulations. In other words, the results provide information about: how hip simulations can be affected by the method used for HJC estimation; which kind of hip movement depends the most/the least on the HJC estimating method; whether the robustness of the HJC estimating methods vary by patients or not; and which methods of HJC estimation have similar/different simulation results.

\section{Methods}

\subsection{MRI acquisition and models reconstruction}

\subsubsection{MRI protocol}

In close collaboration with physicians, a protocol is defined to acquire images carrying sufficient information in a reasonable time. A $1.5 \mathrm{~T}$ Siemens system is used for the acquisition. Due to clinical and technical constraints, high resolution imaging of the complete bones is not applicable. However, the coverage of the full bones is essential to define anatomical coordinate systems [33]. To tackle this issue, fast (3 min approx.) but low resolution acquisitions covering femur and hip bones are first performed. Then high resolution acquisitions, exclusively focused on the joint area, are performed (4 min per hip). This combination of various datasets provides enough information to the segmentation procedure, and avoids excessive acquisition times [15].

\subsubsection{MRI segmentation}

The MRI datasets obtained by the described protocol are segmented to get models of the bones (femur and hip bone) and hip cartilages (femoral cartilage, acetabular cartilage, and labrum). The segmentation method [14, 15, 26] is briefly presented as follows.

Generic models of the bones and cartilages were built once using an interactive segmentation tool. When a new MRI volume needs to be segmented, the generic model is coarsely positioned into the MRI volume. A registration approach, which consists in deforming the generic models to match patient-specific anatomical boundaries, is then adopted. Generic models vertices are considered as lumped mass particles evolving under the Newtonian law of motion. A particle is subjected to internal and external forces in a multi-resolution scheme. Internal forces ensure smoothness and exploit prior knowledge of the models to create constraints (e.g. volume preservation, medial surfaces constraints, and shape priors). External forces use topological (e.g. cartilage-bone attachments), image (maximization of intensity-based similarity measures) and non-penetration (hierarchical collision detection [31] and response [32]) constraints. The first-order differential equation system relating forces to particle state (position and velocity) is resolved by a stable implicit scheme [4, 32]. The segmentation is validated in experiments (in this research, a mean accuracy of $1.5 \mathrm{~mm}$ was reported for the bones segmentation), and the quality of the cartilages segmentation is visually validated by medical experts. Figure 1 shows some examples of segmented 3D models, in which Blue color indicates femoral and acetabular cartilages, while red is used for labrum.

\subsection{HJC estimation methods}

Many different methods of HJC estimation have been proposed that can be classified into predictive and functional approaches. The predictive (static) approach [5, 6, $10,18,27]$ relies upon the location of anatomical landmarks. The functional (dynamic) approach estimates the HJC from recorded [8, 9, 11, 25, 28] or simulated [15-17] motion. It has been reported that the functional approach is more accurate since joint dynamics are taken into account [33]. However, when the movements' amplitude is limited, the predictive approaches can be the only applicable methods (e.g. [7]). Gilles et al. [15] has depicted three predictive and two functional approaches, which we describe here briefly.

\subsubsection{Predictive approaches}

The HJC is estimated as the center of the sphere that approximates the best the femoral head or the acetabulum. The approximation is thus a least square fitting, which aims to find the center (and radius) of the fitted sphere to the reconstructed data. These two methods are denoted as femoralheadsphere and acetabulumsphere methods. They do 

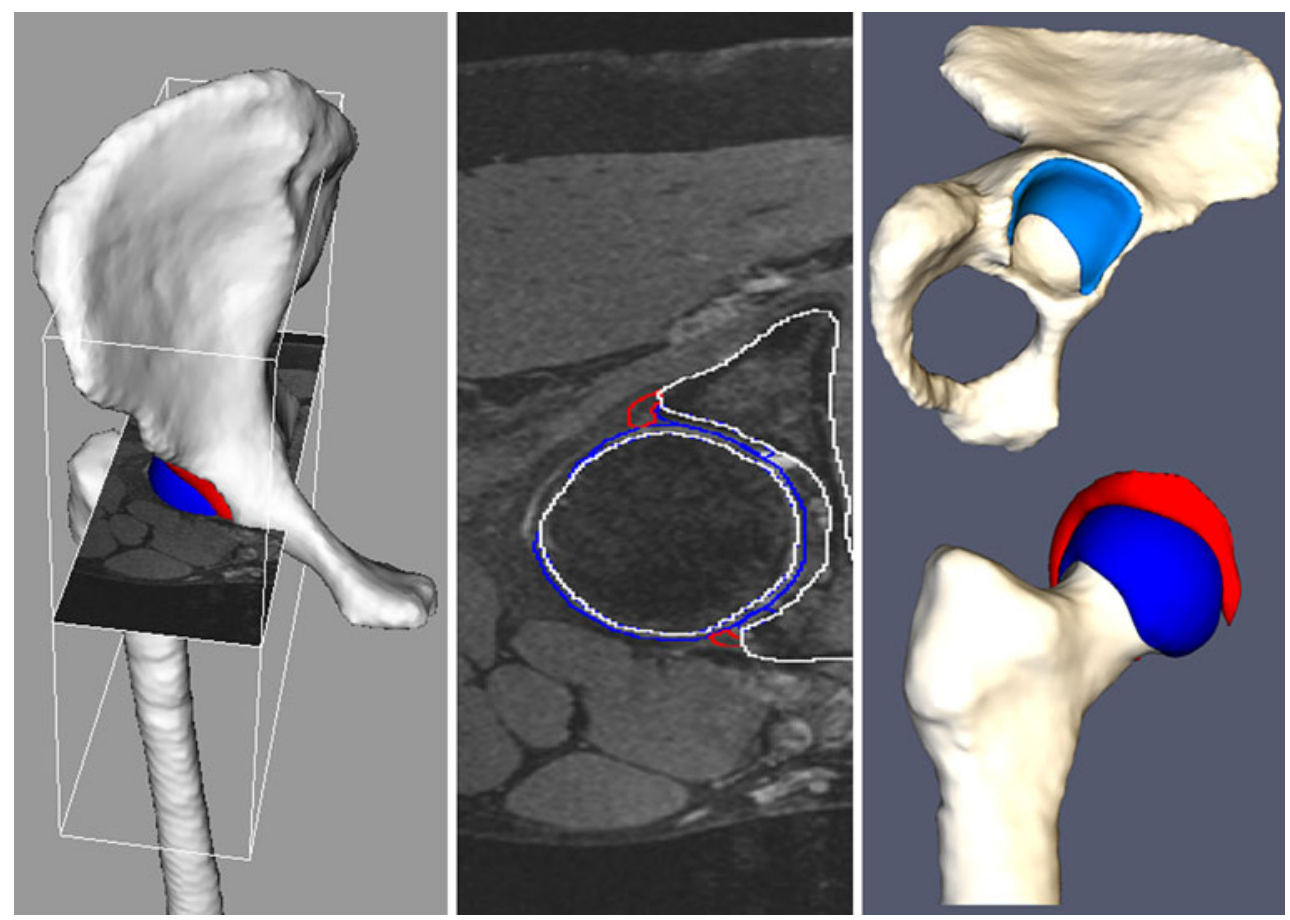

Fig. 1 Left and middle: example of the segmentation overlay in a high resolution dataset focused on the hip joint. Right: example of reconstructed model (right femur and hip bone with femoral cartilage, acetabular cartilage and labrum)

not lead to the same result due to an inhomogeneous interarticular distance. The doublesphere approach considers the joint as a perfect ball and socket joint where interarticular distance is constant. It aims at finding the common center of the femoral and acetabulum spheres (both spheres are centered on the same point). The fitting process for a simple sphere is then extended in this case [15].

\subsubsection{Functional approaches}

The main idea in the functional approach is to enforce a certain inter-articular distance: given each vertex $P_{i}$ of the acetabulum, the difference $\left|d_{i}-d_{i}^{\text {ref }}\right|$ is minimized, where $d_{i}$ $\left(d_{i}^{\text {ref }}\right)$ denotes the (reference) distance between the vertex $P_{i}$ and the femoral head. For a given joint transform, a minimization process is used to minimize the differences $\left|d_{i}-d_{i}^{\text {ref }}\right|$ through hip bone infinitesimal translations. Two different approaches are explored to choose the reference distance: (1) the dconst approach that uses a constant reference distance for all the vertices equal to the radii difference between the fitted femoral and acetabulum spheres; (2) the dref approach that uses the initial distances acquired in the reconstructed position to set the reference distances [15].

In a simulated motion, each joint transform can be hence optimized in terms of shifts to seek reference inter-articular distances. The HJC is then considered as the point of the femur which moves the less in the hip bone frame during the optimized motion. A global minimization technique is thus applied to identify the HJC [15]. The chosen simulated motion is a circumduction pattern [16] with elevation set to $20^{\circ}$.

\subsection{Contact management}

For estimating penetration depth among virtual objects, collision detection methods are used. In fact, collision detection methods return colliding elements (i.e. vertices and polygons building virtual tissues). There are many methods proposed for collision detection, such as methods based on bounding volume hierarchy [20,31], distance fields [31], image-space techniques [3], spatial segmentation [2, 22, 30], average-case approach [19], and random selection method [20]. Arbabi et al. [2] proposed two methods for detecting collision among rotating objects. In these two methods, the penetration depths are calculated in either radial direction (originating from center of rotation) or angular direction (along the circular arc induced by on-going rotation axis). As the methods proposed by Arbabi et al. [2] are specialized for rotating objects, we use them for detecting collision and estimating penetration depth among contacting tissues of the hip joint.

When two objects collide with each other during rotation, two kinds of penetration may occur: (1) tangential or (2) radial. The tangential penetration happens in the angular direction that is tangential to the rotational trajectory (such as when femur bone collides with labrum). On the other hand, the radial penetration usually happens 
among the surfaces that are sliding on each other during rotation (such as when femur cartilage slides under acetabular cartilage) [2]. Therefore, two kinds of penetration are investigated:

\subsubsection{Angular penetration between femur and labrum}

Using the cylindrical segmenting method proposed by Arbabi et al. [2], femur bone is cylindrically segmented and polygon indices are stored in corresponding table cell(s). Then the penetrating labrum vertices are detected by determining the table cell they belong to and checking potential collision with the femur polygons stored in the cell, along a circular trajectory. The penetration depth of a labrum vertex is estimated by calculating the smallest distance that the femur bone needs to be moved away in its rotational trajectory to leave the penetrated vertex of labrum out. Upper part of Fig. 2 illustrates cylindrical segmentation of the space around hip joint and the obtained angular penetration depth. In this illustration, ' $d$ ' represents the amount of angular penetration depth of vertex 'A' located on the labrum (light colored) inside femur bone (dark colored), when the bone is rotating about ' $\mathrm{O}$ '.

\subsubsection{Radial penetration between femoral cartilage and the acetabular soft tissues (consisting of acetabular cartilage and labrum)}

The penetration among femur and acetabular soft tissues is evaluated using Radial Cell-Based Partitioning method

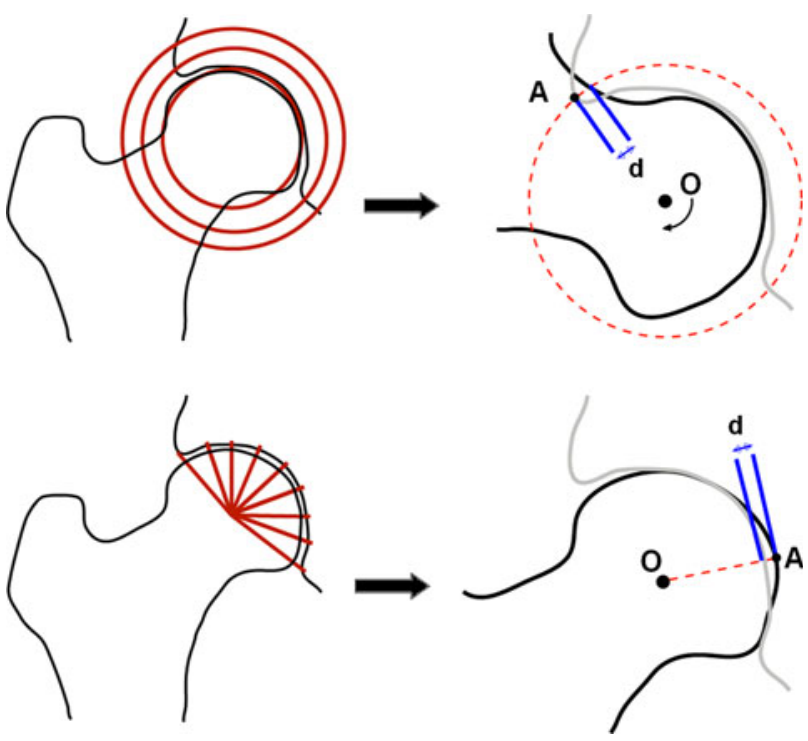

Fig. 2 Upper-left: cylindrical segmentation of the space around the hip joint. Upper-right: angular penetration depth obtained by cylindrical segmenting method. Bottom-left: radial segmentation of the space around the hip joint. Bottom-right: radial penetration depth obtained by Radial Cell-Based Partitioning method proposed by [2]. In this method, instead of applying cylindrical segmentation, the acetabular soft tissues are segmented radially (originating from center of rotation). Therefore, the returning penetration depths are calculated by finding the radial distance between each femur cartilage vertex and the acetabular polygons occupying the same radial segment of the vertex. The method is also repeated by exchanging the role of femur cartilage and acetabular soft tissues. Bottom part of Fig. 2 illustrates radial segmentation of the space around hip joint and the obtained radial penetration depth. In this illustration, ' $d$ ' represents the amount of radial penetration depth of vertex ' $A$ ' located on the femur cartilage (dark colored) inside acetabular cartilage (light colored), when the bone is rotating about ' $\mathrm{O}$ '.

\subsection{Analysis}

Ten healthy female subjects (average age: 24) were selected for the following study. All the experiments were approved by subjects and the ethical committees. After creating 3D meshes of the patients hip tissues based on MR Images, we simulated a patient's hip meshes by rotating the hip about five different HJCs (estimated for each patient, separately) and calculating tissues contact penetration depths (angular and radial). We did these rotations and calculations for all the patients (totally 50 simulations). For simplicity, we name each simulation based on the method used for estimating HJC and the number of patients. For example SIM(doublesphere, 3) stands for the simulation done for the third patient by considering the HJC estimated by doublesphere. In each simulation, we rotated the femur bone about its clinical axes to perform Internal Rotation, External Rotation, Abduction, Adduction, Extension, and Flexion, based on the standards explained by [33]. The maximum amount of rotation was derived from the investigation done by [13] about hip range of motion. For each degree of rotation, we estimated the maximum value of both angular penetration depth (when femur bone collides with labrum) and radial penetration depth (when femoral cartilage slides under the acetabular cartilage and labrum). Therefore, for each patient we obtained the curves showing how the maximum penetration depth changes during rotation about each clinical axis, by considering each of estimated HJC for the corresponding patient. As an example, upper part and bottom part of Fig. 3 are showing maximum amount of radial and angular penetration depths respectively, during hip Extension of a patient. The penetration depths are calculated by applying HJC estimated based on dref (thin solid curve), acetabulumsphere (dotted curve), doublesphere (dashed curve), femoralheadsphere (dot-dashed curve), and dconst (thick solid curve).

For simplicity, we represent the obtained results of each simulation by following arrays: 
Fig. 3 Maximum amount of radial (upper) and angular (bottom) penetration depths during hip Extension of a patient
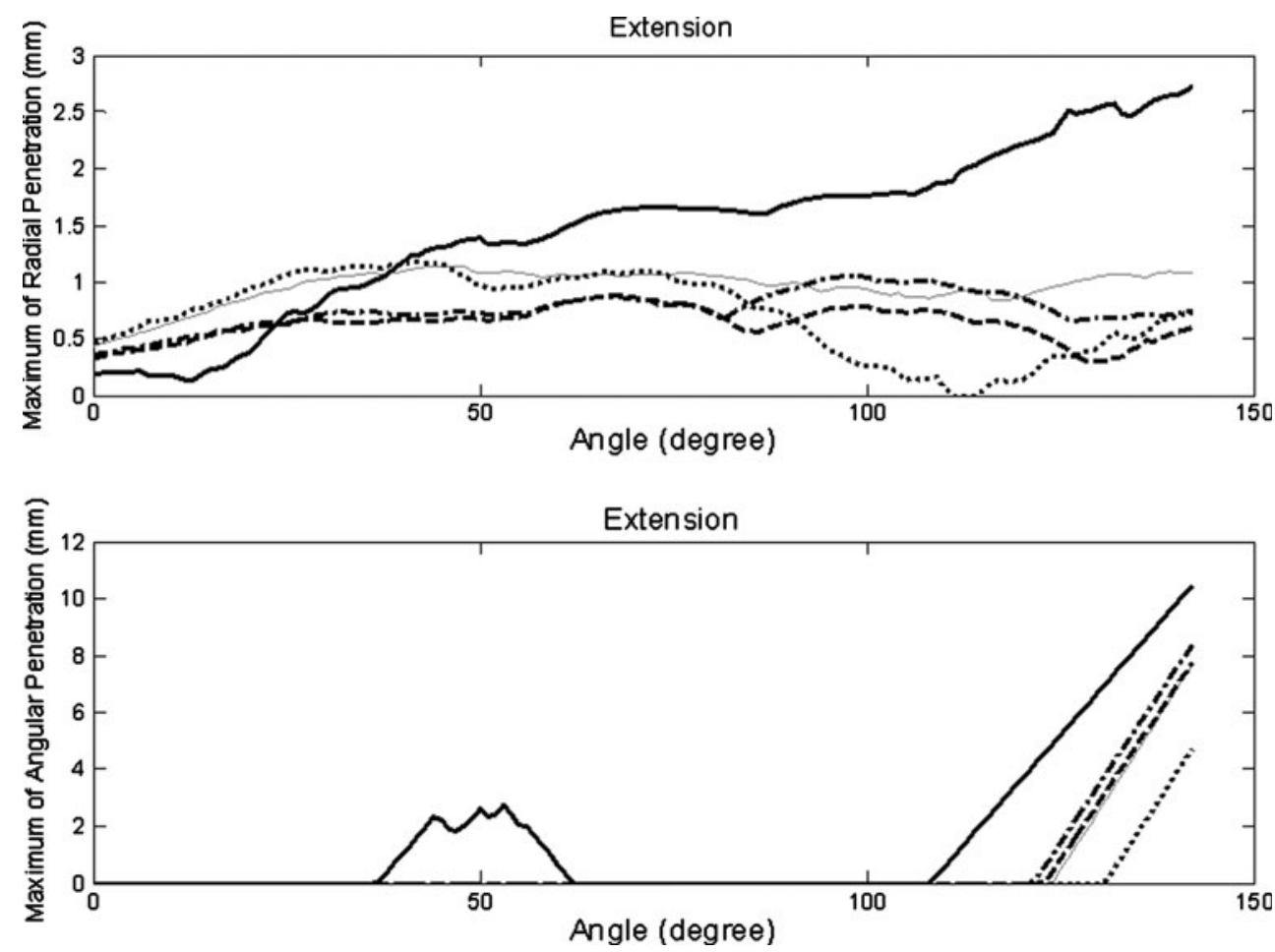

$\operatorname{Array}^{\mathrm{ANG}}=\left[\mathrm{FL}^{\mathrm{ANG}}, \mathrm{EX}^{\mathrm{ANG}}, \mathrm{IR}^{\mathrm{ANG}}, \mathrm{ER}^{\mathrm{ANG}}, \mathrm{AD}^{\mathrm{ANG}}, \mathrm{AB}^{\mathrm{ANG}}\right]$, $\operatorname{Array}^{\mathrm{RAD}}=\left[\mathrm{FL}^{\mathrm{RAD}}, \mathrm{EX}^{\mathrm{RAD}}, \mathrm{IR}^{\mathrm{RAD}}, \mathrm{ER}^{\mathrm{RAD}}, \mathrm{AD}^{\mathrm{RAD}}, \mathrm{AB}^{\mathrm{RAD}}\right]$,

where $\mathrm{FL}^{\mathrm{ANG} / \mathrm{RAD}}, \mathrm{EX}^{\mathrm{ANG} / \mathrm{RAD}}, \mathrm{IR}^{\mathrm{ANG} / \mathrm{RAD}}, \mathrm{ER}^{\mathrm{ANG} / \mathrm{RAD}}$, $\mathrm{AD}^{\mathrm{ANG} / \mathrm{RAD}}$, and $\mathrm{AB}^{\mathrm{ANG} / \mathrm{RAD}}$ correspond to the array of maximum angular/radial penetration depth during Flexion, Extension, Internal Rotation, External Rotation, Adduction, and Abduction, respectively.

\section{Results}

\subsection{Results series 1: per patient}

As a real hip joint does not have any known center of rotation, in order to investigate the effect of HJC in penetration depth, we considered one of the methods for estimating HJC as a reference method and compare the other methods with it. Gilles et al. [15] have presented dref as a method for estimating HJC with smaller error; therefore, we considered the results of SIM(dref, $\mathrm{X}$ ) as our reference values for patient number $X$. It should be noticed that choosing a reference method is just for simplifying the comparison, and having different reference methods returns the same conclusion.

To have a general evaluation, we first considered all the rotations together. Then for each simulation we estimated array of absolute differences in penetration depth (compared to the reference simulation):

$$
\begin{aligned}
& \operatorname{DeltaArray}_{\mathrm{SIM}(i, j)}^{\mathrm{ANG}}=\left|\operatorname{Array}_{\mathrm{SIM}(i, j)}^{\mathrm{ANG}}-\operatorname{Array}_{\mathrm{SIM}(\operatorname{dref}, j)}^{\mathrm{ANG}}\right| \\
& \operatorname{DeltaArray}_{\operatorname{SIM}(i, j)}^{\mathrm{RAD}}=\left|\operatorname{Array}_{\mathrm{SIM}(i, j)}^{\mathrm{RAD}}-\operatorname{Array}_{\mathrm{SIM}(\operatorname{dref}, j)}^{\mathrm{RAD}}\right| \\
& i=\{\text { acetabulumsphere, doublesphere, } \\
& \text { femoralheadsphere, dconst\}; } \\
& j=\{0 \text { to } 10\},
\end{aligned}
$$

where DeltaArray $\mathrm{ANG}_{\mathrm{SIM}(i, j)}^{\mathrm{ANG}}$ and DeltaArray $\mathrm{SIM}_{\mathrm{S}(i, j)}^{\mathrm{RAD}}$ represent the absolute difference between the maximum penetration depths of method $\mathrm{i}$ compared to dref for patient $\mathrm{j}$. In order to have percentage of differences instead of absolute differences, we estimated another array (PercentageDelta$\operatorname{Array} \underset{\operatorname{SIM}(i, j)}{\mathrm{RAD}})$ by dividing each value of DeltaArray $\underset{\operatorname{SIM}(i, j)}{\mathrm{RAD}}$ to its corresponding value in $\operatorname{Array}_{\operatorname{SIM}\left(\mathrm{dref}_{j}, j\right)}^{\mathrm{RAD}}$. Because angular penetration only happens when the femoral bone collides with labrum, its array also included zero (no penetration). Thus, the percentage of differences could not be calculated for angular penetration depths. Finally, mean and standard deviation of each series of DeltaArray $\mathrm{ANG}(i, j)$ and PercentageDeltaArray $\operatorname{RIM}_{\operatorname{SI}(i)}^{\mathrm{RAD}}$ were calculated. Figure 4 (Fig. 5) illustrates maximum difference between radial (angular) penetration depths based on dref HJC estimation and radial (angular) penetration depths based on other estimation methods (upper-left: acetabulumsphere; upper-right: doublesphere; bottom-left: femoralheadsphere; bottom-right: dconst). The values are calculated during all 6 types of clinical movements (all together). The mean values are shown as tick bars, where each bar represents one of the ten 

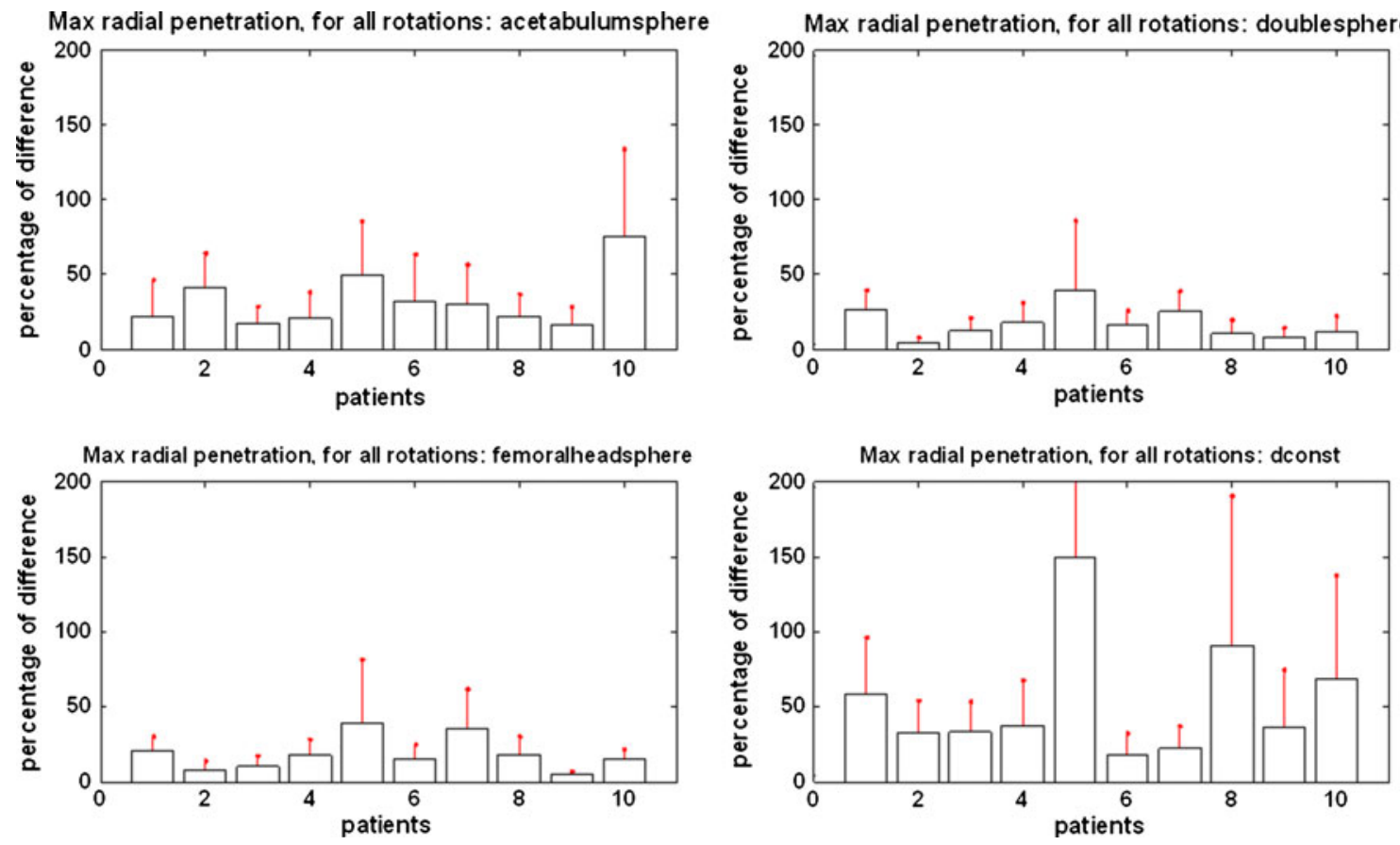

Fig. 4 Maximum difference (in percentage) between radial penetration depths based on dref HJC estimation and radial penetration depths based on other estimation methods (per patient)

patients. The thin line above each bar depicts the standard deviation of the values.

\subsection{Results series 2: per clinical axis of rotation}

In addition to investigating changes in the penetration depth during all the rotations (together), we also investigated the changes in the penetration depth for rotating about each clinical axis separately. In this case, instead of focusing on separated patients, we considered all the patients together and focused on different clinical movements.

By considering (1), we calculated:

$$
\begin{aligned}
\text { DeltaMeanXY } & \mathrm{ANG} \\
\mathrm{SIM}(i, j) & =\operatorname{MEAN}\left(\left|\mathrm{XY}_{\mathrm{SIM}(i, j)}^{\mathrm{ANG}}-\mathrm{XY}_{\mathrm{SIM}(\operatorname{dref}, j)}^{\mathrm{ANG}}\right|\right) \\
\mathrm{XY}= & \{\mathrm{FL}, \mathrm{EX}, \mathrm{IR}, \mathrm{ER}, \mathrm{AD}, \mathrm{AB}\} \\
i= & \{\text { acetabulumsphere, doublesphere, } \\
& \text { femoralheadsphere, dconst }\} ; \\
j= & \{0 \text { to } 10\} .
\end{aligned}
$$

DeltaMeanXY $\mathrm{SIMG}(i, j)$ stands for the mean value of the difference between maximum penetration using type $i$ of HJC and maximum penetration using dref type of HJC, during XY type of clinical movement for the jth patient. By having DeltaMeanXY $\mathrm{SIM}^{\mathrm{ANG} i, j}$ for each patient we created a new array including all the patients together:
DeltaMeanArrayXY $\mathrm{SIM}(i)_{\mathrm{ANG}}=$

$\left[\right.$ DeltaMeanXY $\mathrm{SIM}_{\mathrm{SI}(i, 0)}^{\mathrm{ANG}} ;$ DeltaMeanXY $\mathrm{SIM}_{\operatorname{SI}(i, 1)}^{\mathrm{ANG}} ; \ldots ;$

DeltaMeanXY $\left.\mathrm{SIM}(i, 10)_{\mathrm{ANG}}^{\mathrm{ANG}}\right]$

$i=\{$ acetabulumsphere, doublesphere, femoralheadsphere, dconst\}

The same computations were also done for radial type of penetration. However, instead of calculating absolute differences, we calculated percentage of differences by dividing the absolute differences to the corresponding values when dref method is used. These calculations led us to have PercentageDeltaMeanArrayXY $\operatorname{SIM}_{(i)}^{\mathrm{RAD}}$.

DeltaMeanArrayXY $\mathrm{SIM}_{\mathrm{SI}(i)}^{\mathrm{ANG}}$ and PercentageDeltaMeanArrayXY $\mathrm{SIM}_{\mathrm{SI}(i)}^{\mathrm{RAD}}$ demonstrate how penetration depth differs for method i of HJC estimation compared to the dref method, for a specific clinical movement (XL), by considering all the patients. Thus, we calculated mean, maximum and minimum values of DeltaMeanArrayXY $\mathrm{XY}_{\mathrm{SIM}(i)}^{\mathrm{ANG}}$ and PercentageDeltaMeanArrayXY $\mathrm{XY}_{\operatorname{SIM}(i)}^{\mathrm{RAD}}$. Figure 6 (Fig. 7) illustrates maximum difference between radial (angular) penetration depths based on dref HJC estimation and radial (angular) penetration depths based on the other estimation methods. The values are calculated for all the patients together and during each clinical movement separately (upper-left: Adduction; upper-middle: Abduction; upper-right: Internal Rotation; bottom-left: External Rotation; bottom-middle: Flexion; bottom-right: Extension). 

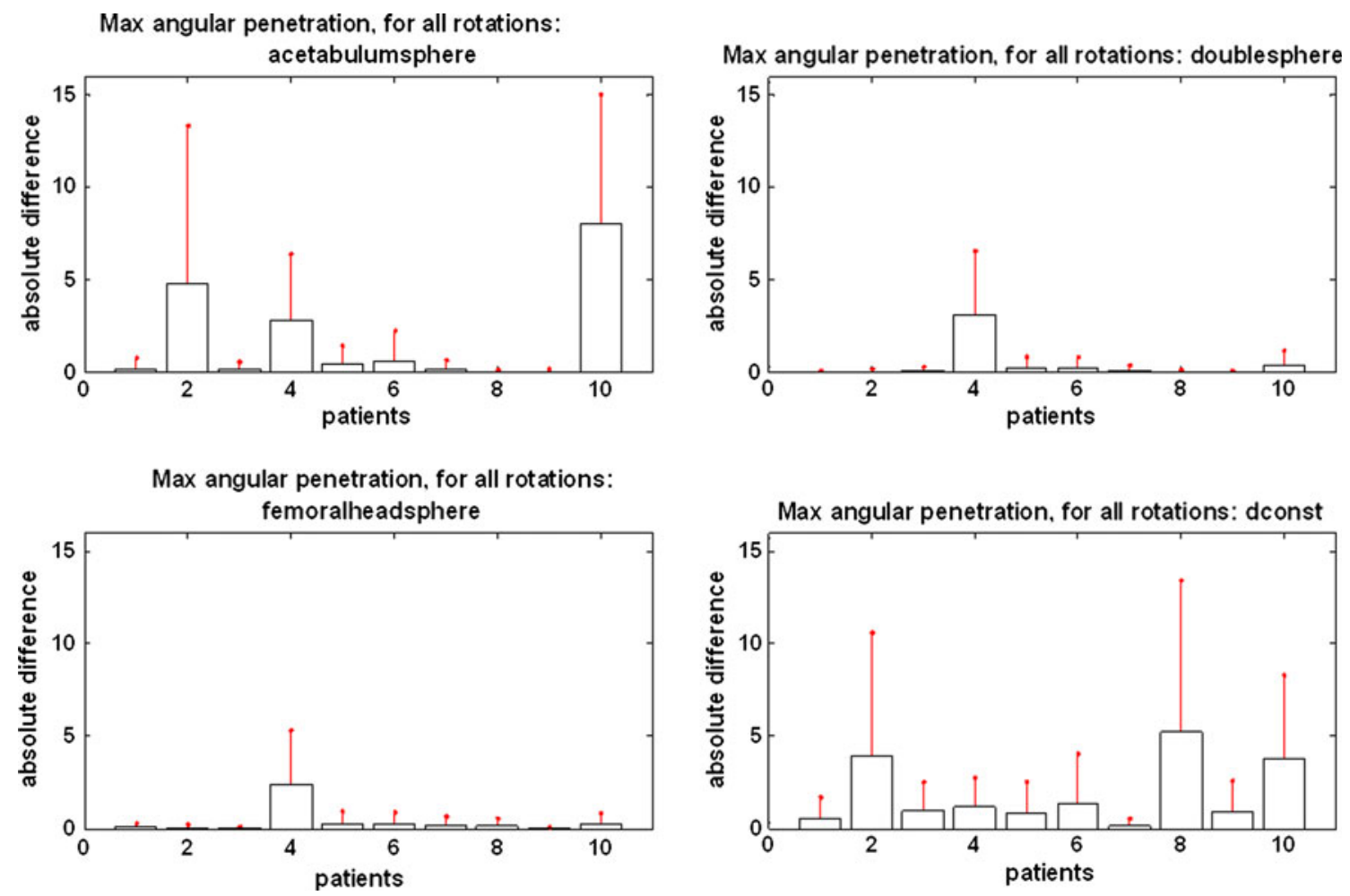

Fig. 5 Maximum difference (in $\mathrm{mm}$ ) between angular penetration depths based on dref HJC estimation and angular penetration depths based on other estimation methods (per patient)

The mean values are shown as a median line of the tick bars, where each bar represents difference between dref method with one of the other methods for estimating HJC (bar \#1: acetabulumsphere; bar \#2: doublesphere; bar \#3: femoralheadsphere; bar\#4: dconst). The T-shape end limit on each bar represents the range of these values (maximum and minimum) (the tick bars are expanded up to $3 / 4$ of their range).

\section{Discussion}

In this paper, we investigated the sensitivity of the penetration depth among hip tissues to the methods applied for estimating the hip joint center of rotation. Our goal was to check this sensitivity during 3D computer-based simulations. Therefore, our estimations were done using 3D meshes reconstructed from MRI rather than using physical models or cadaver [21]. Two different kinds of penetration depths were considered (radial and angular) and the investigations were done for 10 different patients and for 6 types of clinical movements.

When the penetration depths for different patients are investigated separately (results series 1 ), it can be seen that for almost all of the estimated HJCs, radial penetration depth differs considerably (about 30\% in average) compared to when HJC is estimated based on dref. Yet, these differences in estimated penetration depth are not the same for all of HJC estimation methods. The changes in the penetration depth are smaller when doublesphere and femoralheadsphere methods are used for estimating the HJC (less than $19 \%$ in radial case and less than $0.42 \mathrm{~mm}$ in angular case, in average). The differences increase to about $55 \%$ for radial case and $1.87 \mathrm{~mm}$ in angular case (in average) for all the patients when the dconst method is used. Such amount of differences highlights the fact that the hip simulations can give different results and conclusions when the HJC estimation method shifts to one another (especially from dref to dconst). Thus, the medical results of different hip simulations are not comparable if the hip joint center is estimated by different or unknown methods.

These results also show that the difference in penetration depth is patient oriented. For example, results of series 1 show that patient 5 usually has the highest difference in radial penetration depth, when the HJC estimation method changes. Also, patient 4 has the highest difference in angular penetration depth compared to the other patients, when doublesphere and femoralheadsphere methods are applied for estimating HJC. In addition, it demonstrates that radial penetration and angular penetration depth may vary in different manners when the method for estimating HJC changes. 
Fig. 6 Maximum difference (in percentage) between radial penetration depths based on dref HJC estimation and radial penetration depths based on the other estimation methods (per clinical axis of rotation)
Fig. 7 Maximum difference (in $\mathrm{mm}$ ) between angular penetration depths based on dref HJC estimation and angular penetration depths based on the other estimation methods (per clinical axis of rotation)
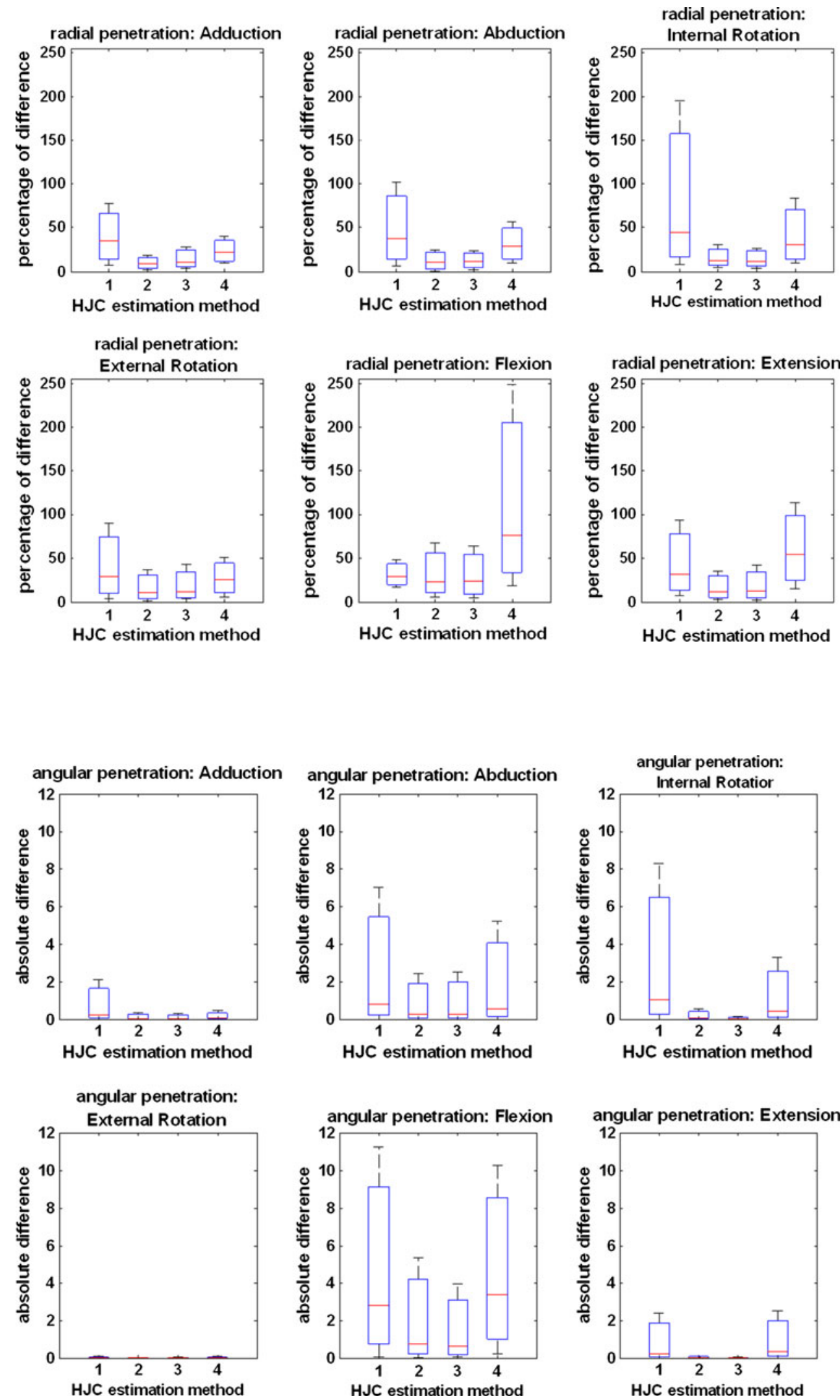
These results suggest that the dconst and acetabulumsphere present the highest variability with respect to the femoralheadsphere and doublesphere methods. This can be explained by various factors. First, the dconst method was depicted by Gilles et al. [15] as the worst method for computing the joint center with respect to the other approaches. Second, the performance of the acetabulumsphere can be explained by errors in the reconstruction of the acetabulum. Indeed, it is more difficult to segment the acetabulum than the femoral head. As a result, the sphere fitting will be affected by these errors. This also explains why the doublesphere method seems to be less sensitive than the acetabulumsphere method, as errors of the acetabulum segmentation are somehow compensated by the higher accuracy in the femoral head reconstruction.

Cartilages are still more difficult to segment compared to bones due to their thickness and their poor contrast in some images. Radiologists often use contrast agent injection to increase the visibility of the cartilages. This operation was not used in our experiments as it remains an invasive procedure. Cartilage segmentation remains hence difficult and resulting errors can as well influence the results. This can explain why some discrepancies exist among the various patients as the segmentation quality can vary from one dataset to another.

When the investigation is focusing on different axis of rotation (results series 2), in almost all of the cases using acetabulumsphere and dconst methods for estimating HJC cause the highest change in both radial and angular penetration. This is consistent with the previous remarks. However, radial and angular penetration depth during Adduction and External Rotation shows to be less sensitive to the methods used for estimating HJC (compared to the other types of rotation). On the other hand, Flexion is among the most sensitive types of rotation to the HJC estimation methods.

This investigation highlights the importance of the HJC estimation methods because of their influence on computer-aided medical researches and diagnosis. In fact, researchers should be careful in choosing the methods of HJC estimation, before providing any conclusion from their medical research. In any case, the HJC estimation methods that should be considered are those that are reported to be more accurate. For example, the dconst method should be avoided. In addition, special attention should be paid in assessing or considering the errors made in the reconstruction as they have an impact on the results. Presented HJC estimation methods are indeed affected by these segmentation errors. Also, the meshes quality and their resolution can affect the accuracy of the penetration depth computation.

Finally, in order to reduce the negative influence of such sensitivity on the hip joint researches, and to have comparable simulation results, a standard method for estimating HJC should be decided and used in all the future researches. For finding the standard method, different kinds of measurement can be used. However, since HJC is affecting the simulations, we suggest calculating the tissue contact penetration depths during the real hip movement (non-idealized) by gradually recording the relative position of the hip tissues, when the patients move their hip in the medical directions. The results should be then compared with the contact penetration depths calculated during idealized hip movement (when the hip joints are rotating about their estimated HJC). A HJC estimation method, which returns the smallest difference in penetration depths for a statistically significant number of patients (compared to the real movement), can be recommended as the standard method.

In conclusion, the results indicate that hip medical investigations are not necessarily robust when the HJC estimation method changes. Particularly, it could be observed that the dconst and acetabulumsphere are the least robust methods in some of the evaluations. In addition, hip simulation results (obtained based on different or unknown HJC estimation method) are more robust and comparable when the hip movement is closer to Adduction or External Rotation, rather than Flexion.

Acknowledgment This research has been supported by the NCCR Co-Me of the Swiss National Science Foundation and by the Marie Curie RTN "3D Anatomical Human" project (MRTN-CT-2006035763) funded by the European Union.

\section{References}

1. Arbabi E, Boulic R, Thalmann D (2007) A fast method for finding range of motion in the human joints. In: Proceedings of 29th Annual International Conference of the IEEE Engineering in Medicine and Biology Society (EMBS'07), Lyon, France, pp 5079-5082

2. Arbabi E, Boulic R, Thalmann D (2009) Fast collision detection methods for joint surfaces. J Biomech 42(2):91-99

3. Baciu G, Wong WS-K, Sun H (1999) Recode: an image-based collision detection algorithm. J Vis Comp Animat 10:181-192

4. Baraff D, Witkin D (1998) Large steps in cloth simulation. In: Proceedings of SIGGRAPH, ACM, pp 43-54

5. Bell A, Petersen D, Brand R (1990) A comparison of the accuracy of several hip center location prediction methods. J Biomech 23:617-621

6. Boudriot U, Hilgert J, Hinrichs F (2006) Determination of the rotational center of the hip. Arch Orthop Trauma Surg 126: 417-420

7. Bush TR, Gutowski PE (2003) An approach for hip joint center calculation for use in seated postures. J Biomech 36(11): $1739-1743$

8. Camomilla V, Cereatti A, Vannozzi G, Cappozzo A (2006) An optimized protocol for hip joint centre determination using the functional method. J Biomech 39:1096-1106

9. Cappozzo A (1984) Gait analysis methodology. Hum Mov Sci $3: 27-54$ 
10. Cerveri P, Lopomo N, Pedotti A, Ferrigno G (2005) Derivation of centers and axes of rotation for wrist and fingers in a hand kinematic model: methods and reliability results. Ann Biomed Eng 33(3):402-412

11. Chang L, Pollard N (2007) Constrained least-squares optimization for robust estimation of center of rotation. J Biomech 40(6):1392-1400

12. France L, Lenoir J, Angelidis A, Meseure P, Cani M-P, Faure F, Chaillou C (2005) A layered model of a virtual human intestine for surgery simulation. Med Images Analysis 9(2):123-132

13. Genoud P, Sadri H, Dora C, Bidaut L, Ganz R, Hoffmeyer P (2000) The hip joint range of motion: a cadaveric study. In: Conference of the European Society of Biomechanics

14. Gilles B, Moccozet L, Magnenat-Thalmann N (2006) Anatomical modelling of the musculoskeletal system from MRI. In: Proceedings of MICCAI, pp 289-296

15. Gilles B, Kolo-Christophe F, Magnenat-Thalmann N, Becker C, Duc S, Menetrey J, Hoffmeyer P (2009) MRI-based assessment of hip joint translations. J Biomech 42(9):1201-1205

16. Kang M, Sadri H, Moccozet L, Magnenat-Thalmann N (2003) Hip joint modeling for the control of the joint center and the range of motions. In: 5th IFAC Symposium on Modeling and Control in Biomedical Systems, pp 20-24

17. Kang MJ (2004) Hip joint center location by fitting conchoid shape to the acetabular rim region of MR images. In: Proceedings of 26th Annual International Conference of the IEEE Engineering in Medicine and Biology Society (EMBS'04), San Francisco, pp 4477-4480

18. Kirkwood R, Culham E, Costigan P (1999) Radiographic and non-invasive determination of the hip joint center location: effect on hip joint moments. Clin Biomech 14:227-235

19. Klein J, Zachmann G (2003) Adb-trees: controlling the error of time-critical collision detection. In: 8th International Fall Workshop Vision, Modeling, and Visualization, pp 19-21

20. Lin MC, Canny JF (1992) Efficient collision detection for animation. In: Proceedings of 3rd Eurographics Workshop on Animation and Simulation

21. Lopomo N, Sun L, Zaffagnini S, Giordano G, Safran MR (2010) Evaluation of formal methods in hip joint center assessment: an in vitro analysis. Clin Biomech 25(3):206-212
22. Maciel A, Boulic R, Thalmann D (2007) Efficient collision detection within deforming spherical sliding contact. IEEE Trans Vis Comp Graph 13(3):518-529

23. Mardones RM, Gonzalez C, Chen Q, Zobitz M, Kaufman KR, Trousdale RT (2005) Surgical treatment of femoroacetabular impingement: evaluation of the effect of the size of the resection. J Bone Joint Surg 87-A:273-279

24. Martin HD (2001) Clinical examination of the hip. Oper Tech Orthop 15:177-181

25. Piazza S, Okita N, Cavanagh P (2001) Accuracy of the functional method of hip joint center location: effects of limited motion and varied implementation. J Biomech 34(7):967-973

26. Schmid J, Magnenat-Thalmann N (2008) MRI bone segmentation using deformable models and shape priors. In: Proceedings of MICCAI, pp 119-126

27. Seidel GK, Marchinda DM, Dijkers M, Soutas-Little RW (1995) Hip joint center location from palpable bony landmarks-a cadaver study. J Biomech 28(8):995-998

28. Siston R, Delp S (2006) Evaluation of a new algorithm to determine the hip joint center. J Biomech 39:125-130

29. Teran J, Sifakis E, Blemker SS, Ng-Thow-Hing V, Lau C, Fedkiw $\mathrm{R}$ (2005) Creating and simulating skeletal muscle from the visible human dataset. IEEE Trans Vis Comp Graph 11(3):317-328

30. Teschner M, Heidelberger B, Mueller M, Pomeranets D, Gross M (2003) Optimized spatial hashing for collision detection of deformable objects. In: Proceedings of Vision, Modeling, Visualization, pp 47-54

31. Teschner M, Kimmerle S, Heidelberger B, Zachmann G, Raghupathi L, Fuhrmann A, Cani M-P, Faure F, Magnenat-Thalmann N, Strasser W, Volino P (2005) Collision detection for deformable objects. Comp Graph Forum 24(1):61-81

32. Volino P, Magnenat-Thalmann N (2000) Implementing fast cloth simulation with collision response. In: Proceedings of International conference of Computer Graphics (CGI), IEEE Computer Society, pp 257-266

33. Wu G, Siegler S, Allard P, Kirtley C, Leardini A, Rosenbaum D, Whittle M, D’Lima D, Cristofolini L, Witte H, Schmid O, Stokes I (2002) ISB recommendation on definitions of joint coordinate system of various joints for the reporting of human joint motionpart I: ankle, hip and spine. J Biomech 35(4):543-548 